\title{
Effect of Behavioral Weight Management Interventions Using Lifestyle mHealth Self-Monitoring on Weight Loss: A Systematic Review and Meta-Analysis
}

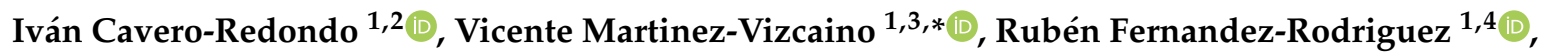 \\ Alicia Saz-Lara ${ }^{1}$ (D), Carlos Pascual-Morena ${ }^{1}$ and Celia Álvarez-Bueno ${ }^{1,2}$ (D) \\ 1 Health and Social Research Center, Universidad de Castilla-La Mancha, 16071 Cuenca, Spain; \\ ivan.cavero@uclm.es (I.C.-R.); ruben.fernandez12@alu.uclm.es (R.F.-R.); Alicia.delsaz@uclm.es (A.S.-L.); \\ carlos.pascual@uclm.es (C.P.-M.); Celia.alvarezbueno@uclm.es (C.Á.-B.) \\ 2 Universidad Politécnica y Artística del Paraguay, Asunción 001518, Paraguay \\ 3 Facultad de Ciencias de la Salud, Universidad Autoónoma de Chile, Talca 3460000, Chile \\ 4 Movi-Fitness S.L, Universidad de Castilla La-Mancha, 16002 Cuenca, Spain \\ * Correspondence: vicente.martinez@uclm.es; Tel.: +34-969-179-100
}

Received: 1 June 2020; Accepted: 30 June 2020; Published: 3 July 2020

\begin{abstract}
Alongside an increase in obesity, society is experiencing the development of substantial technological advances. Interventions that are easily scalable, such as lifestyle (including diet and physical activity) mobile health (mHealth) self-monitoring, may be highly valuable in the prevention and treatment of excess weight. Thus, the aims of this systematic review and meta-analysis were to estimate the following: (i) the effect of behavioral weight management interventions using lifestyle mHealth self-monitoring on weight loss and (ii) the adherence to behavioral weight management interventions using lifestyle mHealth self-monitoring. MEDLINE via PubMed, EMBASE, the Cochrane Central Register of Controlled Trials and the Web of Science databases were systematically searched. The DerSimonian and Laird method was used to estimate the effect of and adherence to behavioral weight management interventions using lifestyle mHealth self-monitoring on weight loss. Twenty studies were included in the systematic review and meta-analysis, yielding a moderate decrease in weight and higher adherence to intervention of behavioral weight management interventions using lifestyle mHealth self-monitoring, which was greater than other interventions. Subgroup analyses showed that smartphones were the most effective mHealth approach to achieve weight management and the effect of behavioral weight management interventions using lifestyle mHealth self-monitoring was more pronounced when compared to usual care and in the short-term (less than six months). Furthermore, behavioral weight management interventions using lifestyle mHealth self-monitoring showed a higher adherence than: (i) recording on paper at any time and (ii) any other intervention at six and twelve months.
\end{abstract}

Keywords: obesity; mHealth; self-monitoring

\section{Introduction}

Overweight and obesity are the fifth highest risk factors for global death, which corresponds to about 3.4 million deaths yearly, making them a global public health priority and a health challenge [1]. According to the World Health Organization (WHO), the obesity rate has tripled since the 1980s. In $2014,39 \%$ of the adult population was classified as overweight and $13 \%$ as obese [2,3], with an 
estimated one billion people classified as overweight and 573 million people as obese [4] in 2030 if there is no attenuation of the current weight gain trends.

The increase in the prevalence of overweight and obesity and their association with many chronic diseases, namely cardiovascular diseases, type 2 diabetes and some cancers, has sparked the interest of researchers and scientific institutions looking for effective ways to promote a healthy lifestyle and weight control [1]. Among behavioral weight loss approaches, lifestyle (including diet and physical activity) and behavior strategies (including self-monitoring) have been consistently related to short- and long-term weight loss management [5] The lifestyle self-monitoring approach consists of registering all food and beverages consumed, portion sizes and methods of preparation, as well as the amount of physical activity performed throughout the day, making individuals aware of their current behaviors [6]. Although, the use of self-monitoring in behavior change has a strong theoretical foundation, completing daily paper records appears to be quite tedious for most individuals [7].

Alongside the increase in obesity, society is experiencing the development of substantial technological advances. While it is true that the boom in new technologies could be involved in the increased availability of energy dense and processed foods as well as the growing prevalence of sedentary behaviors, it is also true that they could play an important role in the management of health problems [8] Interventions based on new technologies could be easily developed and accepted for the management of weight disorders, since they represent an interesting tool to increase individuals' awareness of the quantity and quality of food consumed and the physical activity performed [9] Additionally, lifestyle mobile health (mHealth) self-monitoring [10] appears to have a greater effect on self-efficacy, patient motivation and adherence to treatment, in such a way that it may elicit a greater weight loss than conventional methods [11]. A recent systematic review concluded that mHealth applications may represent an effective strategy for weight loss, and, since the review included both observational and experimental studies, the authors called for an update, including a meta-analysis, when the number of intervention studies allowed for the pooled effect size (ES) of the effect of mHealth applications on weight loss to be estimated [12].

At the present time, considering that obesity prevalence rates do not appear to be decreasing, interventions that are easily scalable, such as lifestyle (diet and physical activity) mHealth self-monitoring, are increasingly valuable in the prevention and treatment of excess weight. However, because no previous meta-analysis has synthesized the effect of lifestyle mHealth self-monitoring as part of behavioral weight management approach, the aims of this systematic review and meta-analysis were to estimate the following: (i) the effect of behavioral weight management interventions using lifestyle mHealth self-monitoring on weight loss and (ii) the adherence to behavioral weight management interventions when lifestyle mHealth self-monitoring was used.

\section{Materials and Methods}

Before conducting this systematic review and meta-analysis, we registered it in the PROSPERO database (registration number ID: CRD42020164608). We followed the Cochrane Handbook for Systematic Reviews of Interventions [13] to conduct it and the Preferred Reporting Items for Systematic Reviews and Meta-Analyses (PRISMA) [14] to report it.

\subsection{Search Strategy}

MEDLINE (via PubMed), EMBASE, the Cochrane Central Register of Controlled Trials and the Web of Science databases were systematically searched, from their inception until March 2020. We searched for experimental studies comparing the effects of behavioral weight management interventions using lifestyle (diet and physical activity) mHealth self-monitoring on weight loss. The search strategy for the MEDLINE database is displayed in Appendix A Table A1. To complete the systematic literature search, we examined the references of the eligible articles. 


\subsection{Study Selection}

The included studies had to meet the following inclusion criteria: (i) participants-general population; (ii) design-randomized controlled trials (RCTs), non-randomized controlled trials (non-RCTs) and pilot studies; (iii) type of interventions-studies comparing the effect of lifestyle (diet and physical activity) mHealth self-monitoring (i.e., personal digital assistants (PDAs), smartphones or web-based); and (iv) outcomes-weight change and adherence to behavioral weight management interventions using lifestyle mHealth self-monitoring. The criteria for the exclusion of studies were as follows: (i) non-eligible publication types, such as review articles, editorials, comments, guidelines or case-reports; and (ii) duplicate reports-when this was the case, we extracted the data from the different reports and included in this systematic review the one providing the most detailed data.

\subsection{Data Extraction and Risk of Bias Assessment}

An ad-hoc table summarized the following information from the original reports: (1) year of publication; (2) country; (3) study design; (4) sample characteristics (sample size, age distribution and type of population); (5) baseline means of adiposity parameters (weight, body mass index (BMI) and waist circumference (WC)); (6) type of intervention (PDA, smartphone or web-based); (7) comparison groups; (8) length of intervention; and (9) percentage of dropouts.

The Cochrane Collaboration's tool for assessing the risk of bias (RoB2) [15] was used to assess the risk of bias of the included RCTs. The evaluation of six domains is included in this tool: randomization process, deviations from intended interventions, missing outcome data, measurement of the outcome and selection of the reported result. Each domain could be assessed as having a low risk of bias, some concerns or a high risk of bias.

For non-RCTs, the ROBINS-I tool was used [16]. This tool evaluates the risk of bias according to seven domains: bias due to confounding, bias in the selection of participants for the study, bias in the measurement of interventions, bias due to deviations from intended interventions, bias due to missing data, bias in the measurement of outcomes and bias in the selection of the reported result. Overall bias could be considered as "low risk of bias" if all domains were classified as "low risk", "moderate risk of bias" if all domains were classified as "low risk" or "moderate risk", "serious risk of bias" if there was at least one domain rated as "serious risk", "critical risk of bias" if there was at least one domain rated as "critical risk" and "no information" if there was no clear indication that the study had a serious or critical risk of bias and there was a lack of information in one or more domains.

The literature search, data extraction and quality assessment were conducted by two independent reviewers (IC-R and RF-R), and a third reviewer (CA-B) was included when inconsistencies remained after discussion. Kappa statistics was calculated to assess the agreement rate between reviewers.

\subsection{Statistical Analysis and Data Synthesis}

To compute the pooled estimate of the ES and its 95\% confidence intervals (CIs) for weight change, we used the DerSimonian and Laird method [17]. A standardized mean difference score was calculated, using Cohen's d index as the ES statistic, in which negative ES values indicate a weight loss in favor of behavioral weight management interventions using lifestyle mHealth self-monitoring. Cohen's d values represented the following: (i) weak effects when values were around 0.2 , (ii) moderate effects when values were around 0.5 , (iii) strong effects when values were around 0.8 and (iv) very strong effects when values were greater than 1.0 [18]. Additionally, a pooled estimate of the mean weight change difference in $\mathrm{kg}$ was calculated.

Adherence to behavioral weight management interventions using lifestyle mHealth self-monitoring was calculated as the risk of dropping out of the lifestyle mHealth self-monitoring group versus other interventions or the control group. Relative risk (RR) was used as the risk estimate.

The heterogeneity of the results across studies was assessed using the $\mathrm{I}^{2}$ statistic. $\mathrm{I}^{2}$ values were interpreted as: might not be important $(0-40 \%)$; may represent moderate heterogeneity $(30-60 \%)$; 
substantial heterogeneity (50-90\%); or considerable heterogeneity $(75-100 \%)$. The corresponding $p$-values were also considered.

Subgroup analyses were performed based on the type of mHealth intervention (PDA, smartphone or web-based), the type of comparison group (usual care, paper record or wait-list) and the length of the intervention ( $\leq 3$ months, six months and $\geq 12$ months). Sensitivity analyses were conducted to assess the robustness of the summary estimates and to detect whether any particular study accounted for a large proportion of heterogeneity. Random-effects meta-regressions were used to investigate whether the results were associated with the age of participants and the baseline means of weight, BMI or WC, since these variables may explain the observed heterogeneity.

Finally, the Egger test [19] ( $p<0.10$ considered as statistically significant [20]) and a visual inspection of the funnel plots were used to assess publication bias. STATA SE software, version 15 (StataCorp, College Station, TX, USA), was used for the statistical analyses.

\section{Results}

\subsection{Systematic Review}

Twenty studies [21-40] (Figure 1) addressing the effect of behavioral weight management interventions using lifestyle mHealth self-monitoring on weight loss were identified, which were conducted in six countries: 12 in the United States [21,23,25,28,31,33-35,37-40], two in the United Kingdom [24,32], three in Australia [22,26,29], one in New Zealand [27], one in South Korea [30] and one in Finland [36]. Reports were published between 2007 and 2019, and they included studies using the following experimental designs: 17 RCTs [21-29,31-38] and three non-RCTs [30,39,40]. Regarding the characteristics of the included populations, participants were aged between 20.5 and 59.8 years, with sample sizes ranging from 11 to 131 participants in the lifestyle mHealth self-monitoring intervention groups and from six to 133 participants in the control groups. The baseline weight, BMI and WC of the studies ranged from $62.1 \mathrm{~kg}$ to $116.9 \mathrm{~kg}$, from $27.0 \mathrm{~kg} / \mathrm{m}^{2}$ to $40.1 \mathrm{~kg} / \mathrm{m}^{2}$ and from $88.2 \mathrm{~cm}$ to $120.4 \mathrm{~cm}$, respectively (Table 1$)$. 
Table 1. Characteristics of studies included in the meta-analysis.

\begin{tabular}{|c|c|c|c|c|c|c|c|}
\hline Reference & Country & Study Design & Mean Age (Years) & Sample Size & $\begin{array}{l}\text { Baseline Weight } \\
(\text { kg Mean } \pm \text { SD) }\end{array}$ & $\begin{array}{c}\text { Baseline BMI } \\
\left(\mathrm{kg} / \mathrm{m}^{2} \text { Mean } \pm \text { SD }\right)\end{array}$ & $\begin{array}{c}\text { Baseline WC } \\
(\mathrm{cm} \text { Mean } \pm \text { SD) }\end{array}$ \\
\hline Allen et al., 2013 [21] & USA & $\mathrm{RCT}$ & $\begin{array}{l}\text { CG: } 42.5 \pm 12.1 \\
\text { IG: } 45.3 \pm 13.2\end{array}$ & $\begin{array}{l}\text { CG: } 18 \\
\text { IG: } 17\end{array}$ & $\begin{array}{l}\text { CG: } 96.0 \pm 17.4 \\
\text { IG: } 96.4 \pm 16.9\end{array}$ & $\begin{array}{c}\text { CG: } 34.1 \pm 4.1 \\
\text { IG: } 35.3 \pm 4.1\end{array}$ & $\begin{array}{c}\text { CG: } 112.4 \pm 11.5 \\
\text { IG: } 109.7 \pm 17.1\end{array}$ \\
\hline Allman-Farinelli et al., 2016 [22] & Australia & RCT & $\begin{array}{c}\text { CG: } 27.2 \pm 4.9 \\
\text { IG: } 28.1 \pm 4.9\end{array}$ & $\begin{array}{l}\text { CG: } 125 \\
\text { IG: } 123\end{array}$ & $\begin{array}{l}\text { CG: } 79.3 \pm 12.6 \\
\text { IG: } 78.4 \pm 11.2\end{array}$ & $\begin{array}{l}\text { CG: } 27.0 \pm 2.7 \\
\text { IG: } 27.3 \pm 2.3\end{array}$ & NR \\
\hline Burke et al., 2011 [23] & USA & $\mathrm{RCT}$ & $\begin{array}{c}\text { CG: } 47.4 \pm 8.5 \\
\text { IG: } 46.7 \pm 9.2\end{array}$ & $\begin{array}{l}\text { CG: } 72 \\
\text { IG: } 68\end{array}$ & NR & $\begin{array}{l}\text { CG: } 33.9 \pm 4.6 \\
\text { IG: } 33.5 \pm 3.8\end{array}$ & $\begin{array}{c}\text { CG: } 109.5 \pm 11.6 \\
\text { IG: } 111.3 \pm 11.1\end{array}$ \\
\hline Carter et al., 2013 [24] & UK & $\mathrm{RCT}$ & $\begin{array}{c}\text { CG: } 42.5 \pm 8.3 \\
\text { IG1: } 41.2 \pm 8.5 \\
\text { IG2: } 41.9 \pm 10.6\end{array}$ & $\begin{array}{l}\text { CG: } 19 \\
\text { IG1: } 40 \\
\text { IG2: } 20\end{array}$ & $\begin{array}{l}\text { CG: } 97.9 \pm 18.7 \\
\text { IG1: } 96.4 \pm 16.0 \\
\text { IG2: } 96.4 \pm 19.9\end{array}$ & $\begin{array}{l}\text { CG: } 34.5 \pm 5.7 \\
\text { IG1: } 33.7 \pm 4.2 \\
\text { IG2: } 34.5 \pm 5.6\end{array}$ & NR \\
\hline Hartman et al., 2016 [25] & USA & RCT & $\begin{array}{c}\text { CG: } 59.8 \pm 5.9 \\
\text { IG: } 59.4 \pm 5.6\end{array}$ & $\begin{array}{l}\text { CG: } 17 \\
\text { IG: } 33\end{array}$ & $\begin{array}{l}\text { CG: } 85.3 \pm 10.5 \\
\text { IG: } 86.3 \pm 10.2\end{array}$ & $\begin{array}{c}\text { CG: } 31.3 \pm 3.7 \\
\text { IG: } 32.2 \pm 3.4\end{array}$ & NR \\
\hline Hutchesson et al., 2018 [26] & Australia & $\mathrm{RCT}$ & $\begin{array}{l}\text { CG: } 27.9 \pm 5 \\
\text { IG: } 27.1 \pm 4.7\end{array}$ & $\begin{array}{l}\text { CG: } 28 \\
\text { IG: } 29\end{array}$ & $\begin{array}{c}\text { CG: } 79.2 \pm 10.3 \\
\text { IG: } 79.8 \pm 10\end{array}$ & $\begin{array}{c}\text { CG: } 29.4 \pm 2.5 \\
\text { IG: } 29.3 \pm 2.5\end{array}$ & $\begin{array}{l}\text { CG: } 88.2 \pm 8.0 \\
\text { IG: } 88.8 \pm 9.0\end{array}$ \\
\hline Jospe et al., 2017 [27] & New Zealand & RCT & $\begin{array}{l}\text { CG: } 46.7 \pm 11.4 \\
\text { IG: } 44.4 \pm 10.2\end{array}$ & $\begin{array}{l}\text { CG: } 36 \\
\text { IG: } 36\end{array}$ & $\begin{array}{l}\text { CG: } 91.0 \pm 14.9 \\
\text { IG: } 99.1 \pm 17.3\end{array}$ & $\begin{array}{c}\text { CG: } 32.3 \pm 4.3 \\
\text { IG: } 33.2 \pm 4.8\end{array}$ & $\begin{array}{l}\text { CG: } 99.8 \pm 11.0 \\
\text { IG: } 102.7 \pm 12.8\end{array}$ \\
\hline Martin et al., 2015 [28] & USA & RCT & $\begin{array}{c}\text { CG: } 43.3 \pm 2.6 \\
\text { IG: } 45.6 \pm 2.7\end{array}$ & $\begin{array}{l}\text { CG: } 20 \\
\text { IG: } 20\end{array}$ & $\begin{array}{c}\text { CG: } 80.6 \pm 2.9 \\
\text { IG: } 80.0 \pm 2.3\end{array}$ & $\begin{array}{c}\text { CG: } 29.5 \pm 3.2 \\
\text { IG: } 30.2 \pm 2.7\end{array}$ & $\begin{array}{l}\text { CG: } 94.5 \pm 2.1 \\
\text { IG: } 93.2 \pm 2.2\end{array}$ \\
\hline Morgan et al., 2013 [29] & Australia & RCT & $\begin{array}{c}\text { CG1: } 48.0 \pm 11.2 \\
\text { CG2: } 48.0 \pm 10.8 \\
\text { IG: } 46.5 \pm 11.1\end{array}$ & $\begin{array}{c}\text { CG1: } 52 \\
\text { CG2: } 54 \\
\text { IG: } 53\end{array}$ & $\begin{array}{c}\text { CG1: } 103.8 \pm 15.0 \\
\text { CG2: } 101.8 \pm 12.4 \\
\text { IG: } 104.7 \pm 14.5\end{array}$ & $\begin{array}{c}\text { CG1: } 33.1 \pm 3.9 \\
\text { CG2: } 32.4 \pm 3.3 \\
\text { IG: } 32.8 \pm 3.4\end{array}$ & $\begin{array}{c}\text { CG1: } 113.6 \pm 9.9 \\
\text { CG2: } 112.6 \pm 9.2 \\
\text { IG: } 113.7 \pm 9.7\end{array}$ \\
\hline Park et al., 2012 [30] & South Korea & Non-RCT & $\begin{array}{c}\text { CG: } 57.6 \pm 5.5 \\
\text { IG: } 55.8 \pm 5.7\end{array}$ & $\begin{array}{l}\text { CG: } 33 \\
\text { IG: } 34\end{array}$ & $\begin{array}{c}\text { CG: } 62.5 \pm 9.0 \\
\text { IG: } 62.1 \pm 7.1\end{array}$ & NR & $\begin{array}{l}\text { CG: } 89.6 \pm 9.9 \\
\text { IG: } 89.9 \pm 5.5\end{array}$ \\
\hline Ross et al., 2016 [31] & USA & $\mathrm{RCT}$ & $\begin{array}{c}\text { CG: } 54.2 \pm 9.5 \\
\text { IG1: } 46.2 \pm 13.5 \\
\text { IG2: } 52.9 \pm 10.3\end{array}$ & $\begin{array}{l}\text { CG: } 26 \\
\text { IG1: } 27 \\
\text { IG2: } 27\end{array}$ & $\begin{array}{l}\text { CG: } 91.6 \pm 14.5 \\
\text { IG1: } 89.2 \pm 15.6 \\
\text { IG2: } 87.1 \pm 12.4\end{array}$ & NR & NR \\
\hline Sniehotta et al., 2019 [32] & UK & RCT & $\begin{array}{l}\text { CG: } 41.6 \pm 11.4 \\
\text { IG: } 42.0 \pm 11.6\end{array}$ & $\begin{array}{l}\text { CG: } 133 \\
\text { IG: } 131\end{array}$ & $\begin{array}{l}\text { CG: } 85.2 \pm 15.7 \\
\text { IG: } 85.1 \pm 17.5\end{array}$ & $\begin{array}{c}\text { CG: } 30.8 \pm 5.2 \\
\text { IG: } 30.9 \pm 5.5\end{array}$ & $\begin{array}{l}\text { CG: } 94.6 \pm 14.7 \\
\text { IG: } 93.6 \pm 13.4\end{array}$ \\
\hline Spring et al., 2013 [33] & USA & RCT & $\begin{array}{l}\text { CG: } 57.7 \pm 10.2 \\
\text { IG: } 57.7 \pm 13.5\end{array}$ & $\begin{array}{l}\text { CG: } 35 \\
\text { IG: } 34\end{array}$ & $\begin{array}{l}\text { CG: } 110.1 \pm 15.1 \\
\text { IG: } 113.7 \pm 16.1\end{array}$ & $\begin{array}{l}\text { CG: } 35.8 \pm 3.8 \\
\text { IG: } 36.9 \pm 5.4\end{array}$ & $\begin{array}{l}\text { CG: } 120.4 \pm 8.9 \\
\text { IG: } 120.4 \pm 14.0\end{array}$ \\
\hline
\end{tabular}


Table 1. Cont.

\begin{tabular}{|c|c|c|c|c|c|c|c|}
\hline Reference & Country & Study Design & Mean Age (Years) & Sample Size & $\begin{array}{l}\text { Baseline Weight } \\
\text { (kg Mean } \pm \text { SD) }\end{array}$ & $\begin{array}{c}\text { Baseline BMI } \\
\left(\mathrm{kg} / \mathrm{m}^{2} \text { Mean } \pm \text { SD) }\right.\end{array}$ & $\begin{array}{c}\text { Baseline WC } \\
(\mathrm{cm} \text { Mean } \pm \text { SD) }\end{array}$ \\
\hline Stephens et al., 2017 [34] & USA & RCT & $\begin{array}{l}\text { CG: } 20.5 \pm 1.7 \\
\text { IG: } 20.8 \pm 2.0\end{array}$ & $\begin{array}{l}\text { CG: } 30 \\
\text { IG: } 29\end{array}$ & $\begin{array}{c}\text { CG: } 79.6 \pm 11.8 \\
\text { IG: } 86.0 \pm 16.3\end{array}$ & $\begin{array}{c}\text { CG: } 29.5 \pm 4.3 \\
\text { IG: } 30.9 \pm 4.4\end{array}$ & $\begin{array}{c}\text { CG: } 97.0 \pm 11.3 \\
\text { IG: } 98.5 \pm 10.8\end{array}$ \\
\hline Svetkey et al., 2015 [35] & USA & $\mathrm{RCT}$ & $\begin{array}{l}\text { CG: } 29.6 \pm 4.3 \\
\text { IG: } 29.2 \pm 4.2\end{array}$ & $\begin{array}{l}\text { CG: } 123 \\
\text { IG: } 122\end{array}$ & NR & $\begin{array}{l}\text { CG: } 35.1 \pm 7.5 \\
\text { IG: } 35.7 \pm 8.2\end{array}$ & NR \\
\hline Teerinemi et al., 2018 [36] & Finland & RCT & $\begin{array}{c}\text { CG: } 46.5 \pm 10.2 \\
\text { IG: } 47.0 \pm 9.4\end{array}$ & $\begin{array}{l}\text { CG: } 59 \\
\text { IG: } 70\end{array}$ & $\begin{array}{c}\text { CG: } 88.6 \pm 11.1 \\
\text { IG: } 88.7 \pm 10.9\end{array}$ & $\begin{array}{l}\text { CG: } 30.5 \pm 2.3 \\
\text { IG: } 30.3 \pm 2.0\end{array}$ & NR \\
\hline Thomas et al., 2019 [37] & USA & $\mathrm{RCT}$ & $55.1 \pm 9.9$ & $\begin{array}{l}\text { CG: } 56 \\
\text { IG: } 114\end{array}$ & $95.9 \pm 17.0$ & $35.2 \pm 5.0$ & NR \\
\hline Wang et al., 2018 [38] & USA & $\mathrm{RCT}$ & $\begin{array}{c}\text { CG1: } 49.2 \pm 10.2 \\
\text { CG2: } 56.1 \pm 5.4 \\
\text { IG: } 58.8 \pm 5.9\end{array}$ & $\begin{array}{l}\text { CG1: } 6 \\
\text { CG2: } 9 \\
\text { IG: } 11\end{array}$ & $\begin{array}{c}\text { CG1: } 92.1 \pm 2.4 \\
\text { CG2: } 116.9 \pm 13.1 \\
\text { IG: } 106.9 \pm 15.1\end{array}$ & $\begin{array}{c}\text { CG1: } 33.7 \pm 2.7 \\
\text { CG2: } 40.1 \pm 7.0 \\
\text { IG: } 38.9 \pm 9.0\end{array}$ & NR \\
\hline Wharton et al., 2014 [39] & USA & Non-RCT & $\begin{array}{c}\text { CG: } 40.8 \pm 3.8 \\
\text { IG1: } 43.7 \pm 3.5 \\
\text { IG2: } 41.5 \pm 4\end{array}$ & $\begin{array}{l}\text { CG: } 20 \\
\text { IG1: } 19 \\
\text { IG2: } 18\end{array}$ & $\begin{array}{l}\text { CG: } 82.2 \pm 20.3 \\
\text { IG1: } 84.2 \pm 13.4 \\
\text { IG2: } 86.1 \pm 22.3\end{array}$ & $\begin{array}{l}\text { CG: } 28.9 \pm 1.0 \\
\text { IG1: } 29.9 \pm 0.9 \\
\text { IG2: } 31.0 \pm 1.7\end{array}$ & NR \\
\hline Yon et al., 2007 [40] & USA & Non-RCT & $\begin{array}{c}\text { CG: } 46.1 \pm 9.2 \\
\text { IG: } 48.2 \pm 8.7\end{array}$ & $\begin{array}{l}\text { CG:93 } \\
\text { IG: } 57\end{array}$ & $\begin{array}{l}\text { CG: } 86.4 \pm 13.7 \\
\text { IG: } 90.2 \pm 14.0\end{array}$ & $\begin{array}{c}\text { CG: } 30.9 \pm 3.5 \\
\text { IG: } 32.3 \pm 3.4\end{array}$ & NR \\
\hline
\end{tabular}

USA: United States of America; UK: United Kingdom; RCT: randomized control trials; CG: Control group; IG: intervention group; NR: not reported; BMI: body mass index; WC:

waist circumference. 


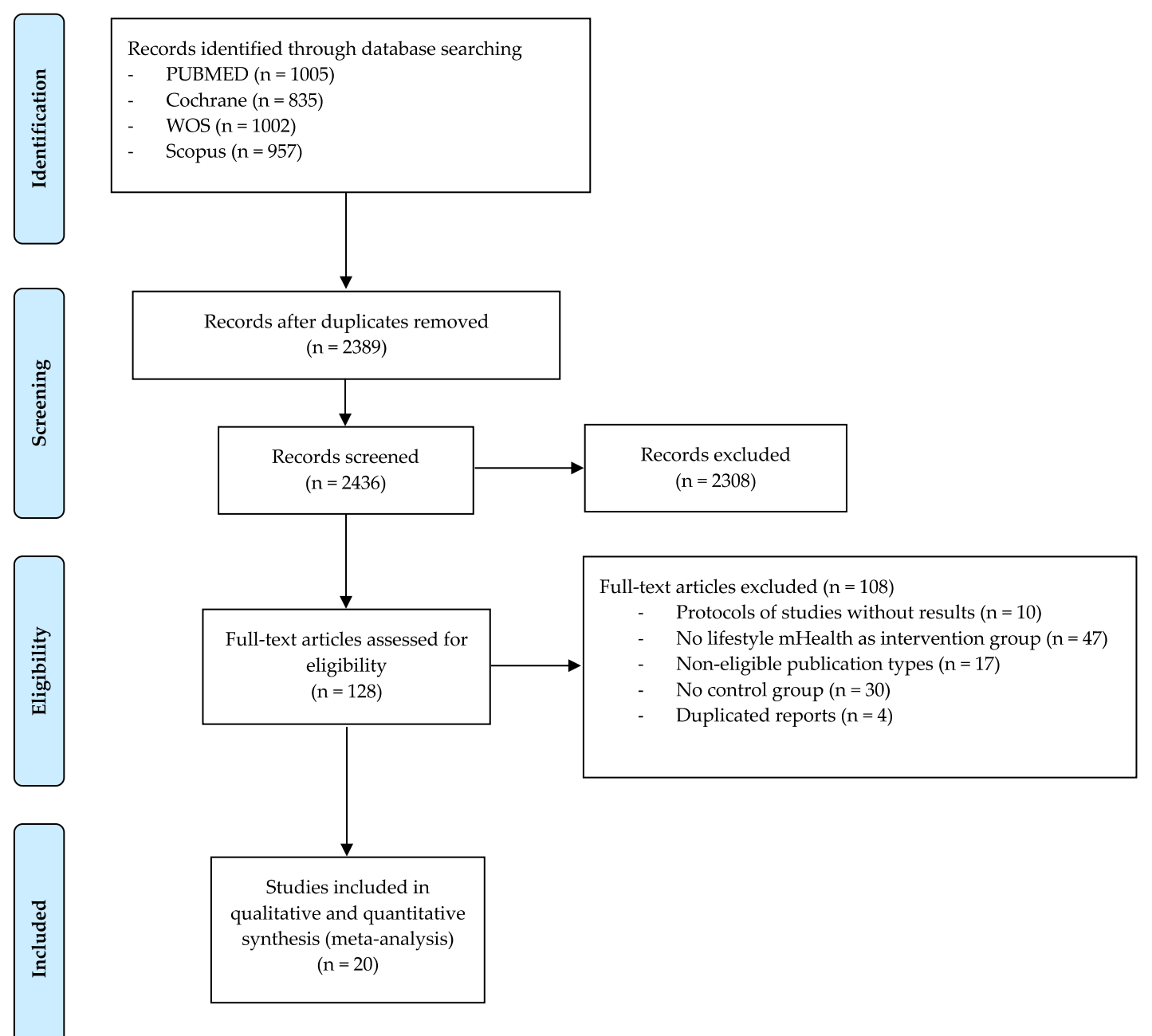

Figure 1. Preferred Reporting Items for Systematic Reviews flowchart.

The mHealth interventions were delivered through PDAs, smartphones and web-based approaches, while comparator groups included usual care, paper records and wait-lists. The length of the interventions ranged from one to 24 months. Eight studies performed analyses for more than one time point $[24,27,28,33,35-38]$. Three studies included two intervention arms [24,31,39]. Additionally, 16 studies specified the application used for lifestyle mHealth self-monitoring: Lose It! [21,34,38,39], TXT2BFiT [22], Dietmate Pro [23], My Meal Mate [24], MyFitnessPal [25,27,37], Be Positive Be Healthy [26], SmartLoss [27], CalorieKing [28,34,39] and Fitbit [30]. The percentage of dropouts from the lifestyle mHealth self-monitoring intervention group ranged from $5.0 \%$ to $54.8 \%$ (Table 2). 
Table 2. Characteristics of type of interventions in the meta-analysis.

\begin{tabular}{|c|c|c|c|c|}
\hline Reference & Intervention & Comparison & Length (Months) & Dropouts (\%) \\
\hline Allen et al., 2013 [21] & Smartphone (Lose It!) & Usual care & 6 & $\begin{array}{l}\text { CG: } 33.3 \\
\text { IG: } 41.2\end{array}$ \\
\hline Allman-Farinelli et al., 2016 [22] & Web-based (TXT2BFiT) & Usual care & 9 & $\begin{array}{l}\text { CG: } 14.4 \\
\text { IG: } 12.8\end{array}$ \\
\hline Burke et al., 2011 [23] & PDA (Dietmate Pro) & Paper record & 6 & $\begin{array}{c}\text { CG: } 12.5 \\
\text { IG: } 5.9\end{array}$ \\
\hline Carter et al., 2013 [24] & $\begin{array}{l}\text { IG1: Smartphone (My Meal Mate) } \\
\text { IG2: Web-based }\end{array}$ & Paper record & 1.5 and 6 & $\begin{array}{c}\text { 6-week follow-up: } \\
\text { CG: } 34.9 \\
\text { IG1: } 9.3 \\
\text { IG2: } 35.7 \\
\text { 6-month follow-up: } \\
\text { CG: } 53.5 \\
\text { IG1: } 7.0 \\
\text { IG2: } 54.8\end{array}$ \\
\hline Hartman et al., 2016 [25] & Smartphone (MyFitnessPal) & Usual care & 6 & $\begin{array}{l}\text { CG: } 5.6 \\
\text { IG: } 8.3\end{array}$ \\
\hline Hutchesson et al., 2018 [26] & Smartphone (Be Positive Be Healthy) & Wait-list & 6 & $\begin{array}{l}\text { CG: } 25.0 \\
\text { IG: } 24.1\end{array}$ \\
\hline Jospe et al., 2017 [27] & Smartphone (MyFitnessPal) & Usual care & 6 and 12 & $\begin{array}{l}\text { 6-month follow-up: } \\
\text { CG: } 8.3 \\
\text { IG: } 20.0 \\
\text { 12-month follow-up: } \\
\text { CG: } 25.0 \\
\text { IG: } 28.0\end{array}$ \\
\hline Martin et al., 2015 [28] & Smartphone (SmartLoss) & Usual care & 1,2 and 3 & $\begin{array}{l}\text { CG: } 5.0 \\
\text { IG: } 5.0\end{array}$ \\
\hline
\end{tabular}


Table 2. Cont

\begin{tabular}{|c|c|c|c|c|}
\hline Reference & Intervention & Comparison & Length (Months) & Dropouts (\%) \\
\hline Morgan et al., 2013 [29] & Web-based (CalorieKing) & $\begin{array}{l}\text { CG1: Wait-list } \\
\text { CG2: Usual care }\end{array}$ & 3 & $\begin{array}{c}\text { CG1: } 7.7 \\
\text { CG1: } 9.2 \\
\text { IG: } 9.4\end{array}$ \\
\hline Park et al., 2012 [30] & Web-based & Wait-list & 3 & NR \\
\hline Ross et al., 2016 [31] & $\begin{array}{l}\text { IG1: Smartphone (Fitbit) } \\
\text { IG2: Smartphone (Fitbit) + phone call }\end{array}$ & Paper record & 6 & $\begin{array}{c}\text { CG: } 11.5 \\
\text { IG1: } 7.4 \\
\text { IG2: } 11.1\end{array}$ \\
\hline Sniehotta et al., 2019 [32] & Web-based & Usual care & 12 & $\begin{array}{l}\text { CG: } 7.6 \\
\text { IG: } 9.0\end{array}$ \\
\hline Spring et al., 2013 [33] & PDA & Usual care & 3,6 and 9 & $\begin{array}{l}\text { 3-month follow-up: } \\
\text { CG: } 14.3 \\
\text { IG: } 11.8 \\
\text { 6-month follow-up: } \\
\text { CG: } 20.0 \\
\text { IG: } 14.7 \\
\text { 9-month follow-up: } \\
\text { CG: } 17.1 \\
\text { IG: } 20.6\end{array}$ \\
\hline Stephens et al., 2017 [34] & Smartphone (LoseIt!) & Usual care & 3 & $\begin{array}{l}\text { CG: } 3.2 \\
\text { IG: } 6.5\end{array}$ \\
\hline Svetkey et al., 2015 [35] & Smartphone (CalorieKing) & Wait-list & 6,12 and 24 & $\begin{array}{l}\text { 6-month follow-up: } \\
\text { CG: } 15.4 \\
\text { IG: } 5.7 \\
\text { 12-month follow-up: } \\
\text { CG: } 13.8 \\
\text { IG: } 10.7 \\
\text { 24-month follow-up: } \\
\text { CG: } 14.6 \\
\text { IG: } 14.8\end{array}$ \\
\hline
\end{tabular}


Table 2. Cont

\begin{tabular}{|c|c|c|c|c|}
\hline Reference & Intervention & Comparison & Length (Months) & Dropouts (\%) \\
\hline Teerinemi et al., 2018 [36] & Web-based & Usual care & 12 and 24 & $\begin{array}{l}\text { 12-month follow-up: } \\
\text { CG: } 23.6 \\
\text { IG: } 13.2 \\
\text { 24-month follow-up: } \\
\text { CG: } 10.1 \\
\text { IG: } 9.9\end{array}$ \\
\hline Thomas et al., 2019 [37] & Smartphone (MyFitnessPal) & Paper record & 6,12 and 18 & $\begin{array}{l}\text { 6-month follow-up: } \\
\text { CG: } 14.3 \\
\text { IG: } 7.9 \\
\text { 12-month follow-up: } \\
\text { CG: } 28.6 \\
\text { IG: } 22.8 \\
\text { 18-month follow-up: } \\
\text { CG: } 33.9 \\
\text { IG: } 19.3\end{array}$ \\
\hline Wang et al., 2018 [38] & Smartphone (LoseIt!) & $\begin{array}{l}\text { CG1: Usual care } \\
\text { CG2: Paper } \\
\text { record }\end{array}$ & 3 and 6 & NR \\
\hline Wharton et al., 2014 [39] & $\begin{array}{l}\text { IG1: Smartphone (LoseIt!) } \\
\text { IG2: Smartphone (Memo function of } \\
\text { the smartphone) }\end{array}$ & Paper record & 2 & NR \\
\hline Yon et al., 2007 [40] & $\begin{array}{l}\text { PDA (Calorie King's Handheld Diet } \\
\text { Diary) }\end{array}$ & Paper record & 6 & $\begin{array}{l}\text { CG: } 19.0 \\
\text { IG: } 7.0\end{array}$ \\
\hline
\end{tabular}

CG: Control group; IG: intervention group; PDA: personal digital assistant; NR: not reported. 


\subsection{Risk of Bias}

For RCTs, as evaluated by the RoB2 tool, $47.4 \%$ of studies showed some concerns regarding the risk of bias and $52.6 \%$ showed a high risk for overall bias (mainly as a consequence of a high risk of bias in the measurement of the outcome domain) (see Appendix A Figure A1). Among non-RCTs, as evaluated by the ROBINS-I tool, the risk of bias was scored as moderate in $33.3 \%$ of studies and serious in $66.7 \%$ (mainly as a consequence of a serious risk of bias in the missing data domain) (see Appendix A Figure A2).

\subsection{Meta-Analysis}

The pooled ES of behavioral weight management interventions using lifestyle mHealth self-monitoring on weight loss was -0.37 ( $95 \% \mathrm{CI}$ : $-0.54,-0.19)$. Additionally, the pooled mean difference in weight was $-1.78 \mathrm{~kg}(95 \% \mathrm{CI}:-2.70,-0.85)$. The heterogeneity between studies was substantial (I2 $=84.6 \% ; p<0.001$ ) (Figure 2). The pooled RR for dropping out of the lifestyle mHealth self-monitoring group was 0.78 (95\%CI: $0.63,0.96)$. The heterogeneity between studies was moderate (I2 $=37.8 \% ; p=0.049)$ (Figure 3).

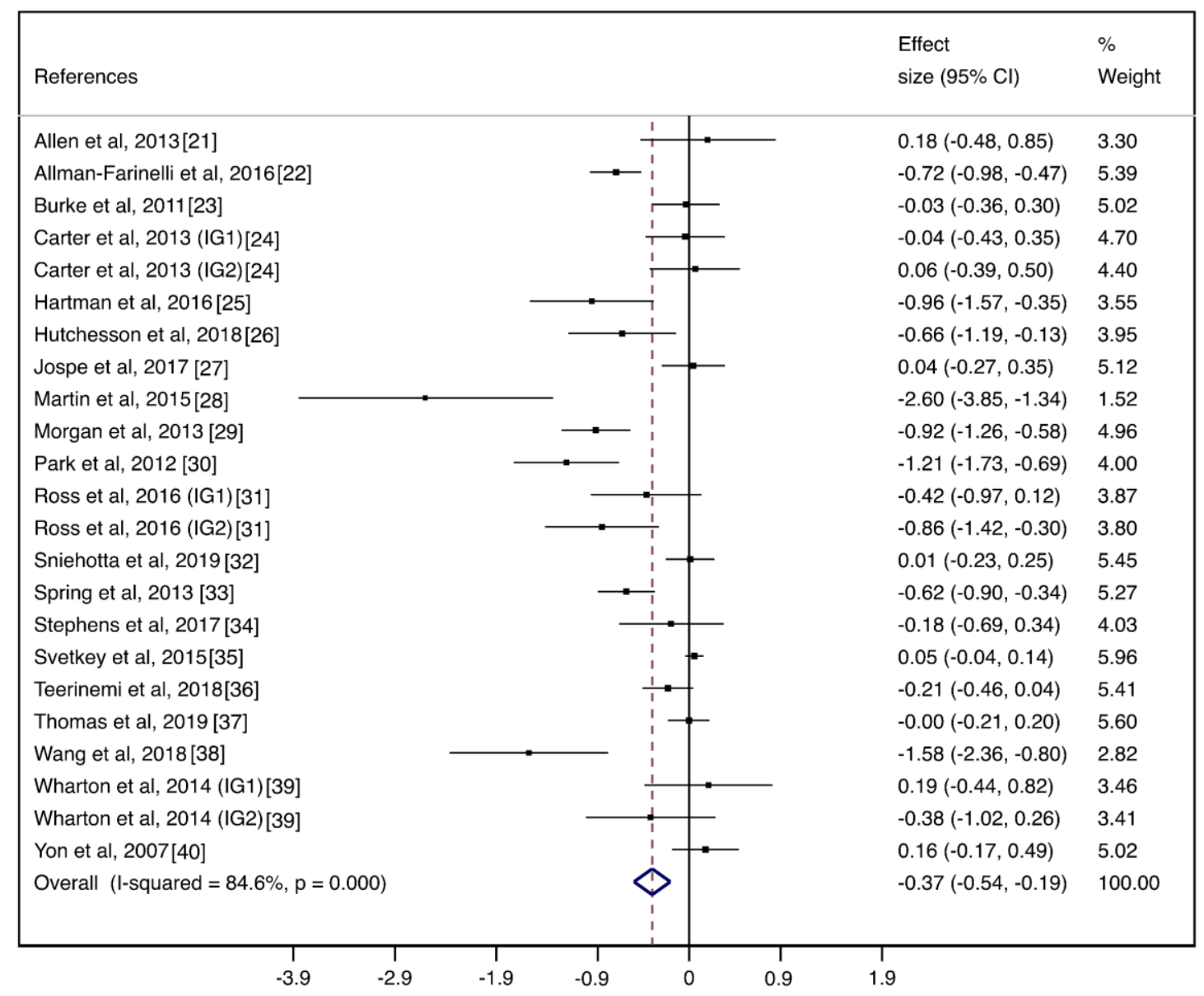

Figure 2. Forest plots of the pooled effect size for behavioral weight management interventions using lifestyle mHealth self-monitoring on weight loss. 


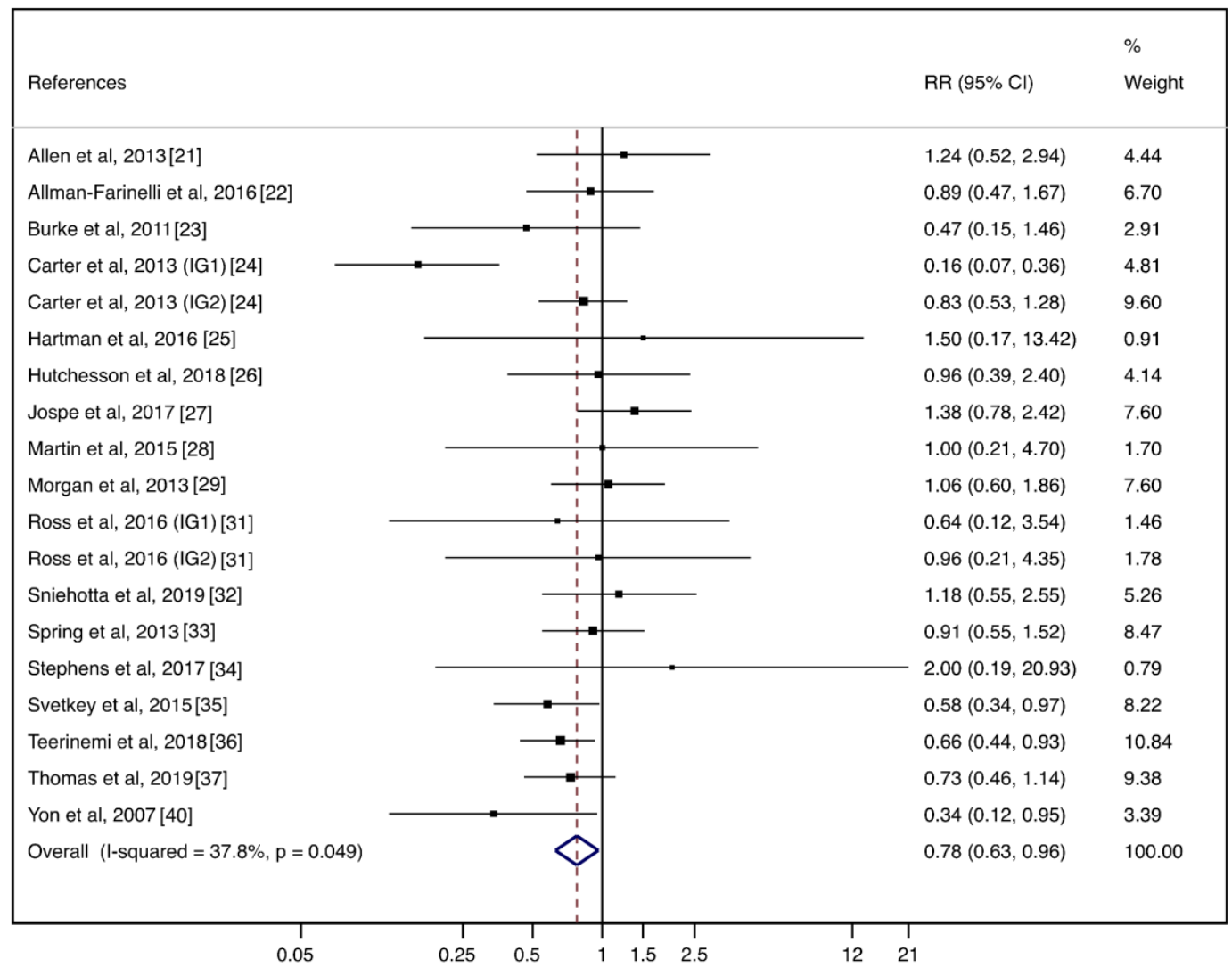

Figure 3. Forest plots of the pooled relative risk for the adherence of behavioral weight management interventions using lifestyle mHealth self-monitoring.

\subsection{Subgroup Analyses}

Subgroup analyses considering the type of mHealth intervention and the type of comparison group showed that a greater effect was observed when the mHealth intervention used a smartphone $(\mathrm{ES}=-0.36 ; 95 \% \mathrm{CI}:-0.51,-0.13, \mathrm{I} 2=56.4 \%)$ and usual care was the control group (ES $=-0.51 ; 95 \% \mathrm{CI}$ : $-0.83,-0.20, \mathrm{I} 2=84.0 \%)$. Additionally, based on the length of the intervention, a pooled ES for a weight loss of -1.08 (95\%CI: $-1.55,-0.62, \mathrm{I} 2=87.6 \%)$ was estimated for $\leq 3$ months and of $-0.23(95 \% \mathrm{CI}$ : $-0.49,-0.02, \mathrm{I} 2=70.4 \%$ ) for six months. Regarding adherence, there were less dropouts in mHealth interventions when paper records were used for the control group $(R R=0.63 ; 95 \% C I$ : $0.44,0.91$, I2 = $20.9 \%)$. Additionally, there were less dropouts from the mHealth interventions in studies performed for six months ( $R R=0.76$; $95 \% \mathrm{CI}: 0.59,0.97, \mathrm{I} 2=46.0 \%$ ) and for twelve months (RR $=0.79 ; 95 \% \mathrm{CI}: 0.64$, $0.96, \mathrm{I} 2=0.0 \%$ ) (Table 3).

Table 3. Subgroup analyses for pooled effect on weight loss and adherence to mHealth group according to type of mHealth intervention, type of comparison and length of intervention.

\begin{tabular}{|c|c|c|c|c|c|c|c|c|}
\hline \multirow[b]{2}{*}{ Subgroup } & \multicolumn{4}{|c|}{ Effect on Weight Loss } & \multicolumn{4}{|c|}{ Adherence to mHealth } \\
\hline & $n$ & $\begin{array}{c}\text { Effect Size } \\
(95 \% \mathrm{CI})\end{array}$ & I2 & $p$ & $n$ & $\begin{array}{l}\text { Relative Risk } \\
\quad(95 \% \mathrm{CI})\end{array}$ & I2 & $p$ \\
\hline \multicolumn{9}{|c|}{ Type of mHealth intervention } \\
\hline Smartphone & 14 & $\begin{array}{c}-0.36 \\
(-0.58,-0.13)\end{array}$ & 56.4 & 0.005 & 11 & $\begin{array}{c}0.76 \\
(0.51,1.16)\end{array}$ & 54.5 & 0.015 \\
\hline PDA & 4 & $\begin{array}{c}-0.17 \\
(-0.46,0.13)\end{array}$ & 62.7 & 0.045 & 3 & $\begin{array}{c}0.61 \\
(0.32,1.15)\end{array}$ & 40.6 & 0.186 \\
\hline Web-based & 2 & $\begin{array}{c}0.02 \\
(-0.21,0.24)\end{array}$ & 0.0 & 0.838 & 5 & $\begin{array}{c}0.83 \\
(0.66,1.04)\end{array}$ & 0.0 & 0.556 \\
\hline
\end{tabular}


Table 3. Cont.

\begin{tabular}{|c|c|c|c|c|c|c|c|c|}
\hline \multirow[b]{2}{*}{ Subgroup } & \multicolumn{4}{|c|}{ Effect on Weight Loss } & \multicolumn{4}{|c|}{ Adherence to mHealth } \\
\hline & $n$ & $\begin{array}{l}\text { Effect Size } \\
(95 \% \mathrm{CI})\end{array}$ & I2 & $p$ & $n$ & $\begin{array}{l}\text { Relative Risk } \\
\quad(95 \% \mathrm{CI})\end{array}$ & I2 & $p$ \\
\hline \multicolumn{9}{|c|}{ Type of Comparison } \\
\hline Usual care & 10 & $\begin{array}{c}-0.51 \\
(-0.83,-0.20)\end{array}$ & 84.0 & $<0.001$ & 10 & $\begin{array}{c}0.97 \\
(0.72,1.30)\end{array}$ & 0.0 & 0.677 \\
\hline Paper record & 11 & $\begin{array}{c}-0.22 \\
(-0.46,0.02)\end{array}$ & 68.4 & $<0.001$ & 7 & $\begin{array}{c}0.63 \\
(0.44,0.91)\end{array}$ & 20.9 & 0.270 \\
\hline Wait-list & 4 & $\begin{array}{c}-0.56 \\
(-1.14,0.01)\end{array}$ & 94.1 & $<0.001$ & 3 & $\begin{array}{c}0.93 \\
(0.59,1.48)\end{array}$ & 0.0 & 0.697 \\
\hline \multicolumn{9}{|c|}{ Length of Intervention } \\
\hline$\leq 3$ months & 14 & $\begin{array}{c}-1.08 \\
(-1.55,-0.62)\end{array}$ & 87.6 & $<0.001$ & 7 & $\begin{array}{c}0.91 \\
(0.61,1.35)\end{array}$ & 0.0 & 0.474 \\
\hline Six months & 15 & $\begin{array}{c}-0.23 \\
(-0.44,-0.02)\end{array}$ & 70.4 & $<0.001$ & 15 & $\begin{array}{c}0.76 \\
(0.59,0.97)\end{array}$ & 46.0 & 0.035 \\
\hline$\geq 12$ months & 5 & $\begin{array}{c}0.02 \\
(-0.07,0.11)\end{array}$ & 0.0 & 0.432 & 6 & $\begin{array}{c}0.79 \\
(0.64,0.96)\end{array}$ & 0.0 & 0.530 \\
\hline
\end{tabular}

PDA: Personal digital assistant.

\subsection{Sensitivity Analyses}

The pooled ES estimate was not significantly different when data from each individual study were removed from the analyses one at a time.

\subsection{Meta-Regressions}

The random-effects meta-regression models for the effects on weight loss and adherence showed that age ( $p=0.365$ and 0.462) and baseline means of weight ( $p=0.724$ and 0.593), BMI ( $p=0.440$ and $0.979)$ and WC ( $p=0.677$ and 0.428$)$ were not related to heterogeneity across studies (see Appendix A Table A2).

\subsection{Publication Bias}

Evidence of publication bias was found in both the funnel plot asymmetry and Egger's test ( $p=0.016$ for effect on weight loss and $p=0.008$ for adherence).

\section{Discussion}

This systematic review and meta-analysis provides an overview of the evidence supporting lifestyle (diet and physical activity) mHealth self-monitoring, as part of a behavioral weight management approach, as a suitable intervention for weight management in adults with overweight or obesity, resulting in a moderate decrease in weight and higher adherence to intervention, greater than with other interventions. Additionally, this meta-analysis shows that interventions delivered through smartphones are the most effective mHealth approach to achieve weight management in adult populations with overweight or obesity. The effect of behavioral weight management interventions using lifestyle mHealth self-monitoring interventions was more pronounced when they were compared to usual care and in the short-term (less than six months). Furthermore, behavioral weight management interventions using lifestyle mHealth self-monitoring interventions showed a higher adherence than: (i) paper records at any time and (ii) any other intervention at six months and twelve months.

A previous systematic review on the effect of mHealth applications on weight loss highlighted that the use of these interventions is widely accepted, easy to use and helpful in achieving weight loss goals [12]. Additionally, there is evidence for the consistent and significant positive relationship 
between lifestyle (diet and physical activity) and behavior (self-monitoring) strategies and successful weight management [5]. Likewise, our systematic review and meta-analysis supports the notion that mHealth self-monitoring interventions have a moderate effect on reducing weight, which may represent a mean weight loss of $1.78 \mathrm{~kg}$ greater than with other intervention types.

Our results not only show a positive effect on weight loss but also fewer dropouts of subjects included in mHealth interventions in the short and long-term. The mechanisms through which behavioral weight management interventions using lifestyle mHealth self-monitoring are effective may be explained from two perspectives: the user and the clinical setting [41]. For users, mHealth interventions enhance patients' self-efficacy and empowerment and improve daily life autonomy and adherence to treatment [42]. Moreover, the mHealth approach reduces contact with the clinical setting and, as a consequence, decreases the workload for health care workers (physicians, nurses and nutritionists, especially in primary care) $[43,44]$.

There is a variety of devices that could be used for mHealth. Our subgroup analyses support the notion that smartphones, a technology available to a high proportion of the population worldwide [45], are the most effective mHealth devices for weight management. This, along with the high prevalence rates of both physical inactivity and obesity, has triggered a growing interest in the development of smartphone applications for health, fitness and diet, which have increased exponentially in the last few years [46]. More than half of smartphone users may have downloaded a health application [47]; however, the use of these applications for clinical outcomes is still very limited and even non-existent in most contexts [48].

The limitations of this study are as follows: (1) the risk of bias assessment showed that a few studies presented some concerns or moderate risk of bias, while most showed a high or serious risk of bias. It should be noted that the main reason behind the high risk of bias in the included studies was the impracticality of blinding interventions, but this limitation is difficult to overcome in this type of intervention. (2) The lack of studies using devices that did not allow for comparison between them, with reliable results only being obtained for smartphones. (3) The intervention groups could be very different considering that they used different types of applications; however, they have the common characteristic that they were carried out through an mHealth device. (4) Many of the studies did not control for the effect of other covariates which could affect the results, such as educational or socioeconomic level. (5) Regarding the analysis of adherence, it cannot be assumed that lifestyle mHealth self-monitoring is the only reason for participants to dropout, since these interventions are usually part of a broader behavioral weight management approach. (6) Finally, this meta-analysis showed publication bias, mainly due to the lack of studies with small sample sizes.

Even with the risk of being branded as opportunists, we are not reluctant to emphasize the importance of mHealth interventions in times when face-to-face contact must be limited, such as those we are living in currently, particularly in clinical settings, in which the transmission of infectious diseases, namely Influenza or Sars-CoV-2, may be greater. Additionally, everything appears to indicate that the current concept of treatment will lead to an increase in the use of mHealth in daily clinical practice [49].

\section{Conclusions}

In summary, our study demonstrates that lifestyle mHealth self-monitoring interventions, as part of a behavioral weight management approach, are suitable interventions for short-term weight management in adults with overweight/obesity. Considering our results and the population's accessibility to smartphones, this type of device could be a useful and largely scalable tool for weight management. Thus, future well designed RCTs and controlled clinical trials with higher statistical power are essential in order to reinforce the evidence, which is still weak, to demonstrate that effective mHealth interventions could eventually change the current paradigm of lifestyle prescription, increasing patients' self-management of disease, developing new clinical practice guidelines and facilitating workflow in everyday clinical consultations. 
Author Contributions: Conceptualization, I.C.-R. and R.F.-R.; methodology, I.C.-R. and C.Á.-B.; software, I.C.-R.; validation, V.M.-V. and C.Á.-B.; formal analysis, R.F.-R. and I.C.-R.; investigation, I.C.-R.; resources, A.S.-L. and C.P.-M.; data curation, C.Á.-B. and V.M.-V.; writing-original draft preparation, I.C.-R.; writing-review and editing, C.Á.-B. and V.M.-V.; visualization, A.S.-L. and C.P.-M.; supervision, C.Á.-B. and V.M.-V. All authors have read and agreed to the published version of the manuscript.

Funding: This study was funded by FEDER funds.

Conflicts of Interest: The authors declare no conflict of interest.

\section{Appendix A}

Table A1. Search strategy for the MEDLINE database.

\begin{tabular}{llll}
\hline \multicolumn{1}{c}{ Search Set Medline } & & \multicolumn{1}{c}{ Search Set Medline } \\
\hline$\# 1$ & smartphone technology [All Fields] & $\# 23$ & telemedicine [Mesh Terms] \\
$\# 2$ & smartphone application [All Fields] & $\# 24$ & telemedicine [All Fields] \\
$\# 3$ & electronic self-monitoring [All Fields] & $\# 25$ & ehealth [All Fields] \\
$\# 4$ & self-monitoring technology [All Fields] & $\# 26$ & 23 OR 24 OR 25 \\
$\# 5$ & self-monitoring [All Fields] & $\# 27$ & mobile devices [All Fields] \\
$\# 6$ & dietary self-monitoring [All Fields] & $\# 28$ & mobile [All Fields] \\
$\# 7$ & website [All Fields] & $\# 29$ & connected tools [All Fields] \\
$\# 8$ & technology [Mesh Terms] & $\# 30$ & 1 OR 2 OR 3 OR 4 OR 5 OR 6 OR 7 OR 10 OR 14 OR 15 OR 18 OR 19 OR 20 \\
$\# 9$ & technology [All Fields] & OR 21 OR 22 OR 26 OR 27 OR 28 OR 29 \\
$\# 10$ & 8 OR 9 & $\# 31$ & obesity treatment [All Fields] \\
$\# 11$ & telephone [Mesh Terms] & $\# 32$ & weight loss [All Fields] \\
$\# 12$ & telephone [All Fields] & $\# 33$ & weight advice [All Fields] \\
$\# 13$ & phone [All Fields] & $\# 34$ & weight management [All Fields] \\
$\# 14$ & 11 OR 12 OR 13 & $\# 35$ & 31 OR 32 OR 33 OR 34 \\
$\# 15$ & phone-based [All Fields] & $\# 36$ & randomized controlled pilot study [All Fields] \\
$\# 16$ & smartphone [Mesh Terms] & $\# 37$ & pilot randomized controlled trial [All Fields] \\
$\# 17$ & smartphone [All Fields] & $\# 38$ & randomized pilot study [All Fields] \\
$\# 18$ & 16 OR 17 & $\# 39$ & randomized controlled trial [All Fields] \\
$\# 19$ & smartphone-based [All Fields] & $\# 40$ & RCT [All Fields] \\
$\# 20$ & smartphone app [All Fields] & $\# 41$ & randomized trial [All Fields] \\
$\# 21$ & personal digital assistant [All Fields] & $\# 42$ & 36 OR 37 OR 38 OR 39 OR 40 OR 41 \\
$\# 22$ & PDA [All Fields] & $\# 43$ & 30 AND35 AND 42 \\
\hline
\end{tabular}

Table A2. Random-effects meta-regressions for the effects of age, weight, BMI and WC on the pooled effect on weight loss and adherence to mHealth.

\begin{tabular}{|c|c|c|c|c|c|c|}
\hline & \multicolumn{3}{|c|}{ Effect on Weight Loss } & \multicolumn{3}{|c|}{ Adherence to mHealth } \\
\hline Variable & $n$ & $\begin{array}{l}\text { Coefficient } \\
(95 \% \mathrm{CI})\end{array}$ & $p$ & $n$ & $\begin{array}{l}\text { Coefficient } \\
(95 \% \mathrm{CI})\end{array}$ & $p$ \\
\hline Age (years) & 23 & $\begin{array}{c}-0.01 \\
(-0.04,0.01)\end{array}$ & 0.365 & 19 & $\begin{array}{c}0.01 \\
(-0.02,0.04)\end{array}$ & 0.462 \\
\hline $\begin{array}{l}\text { Baseline mean weight } \\
\qquad(\mathrm{kg})\end{array}$ & 21 & $\begin{array}{c}0.06 \\
(-0.03,0.04)\end{array}$ & 0.724 & 17 & $\begin{array}{c}0.01 \\
(-0.03,0.04)\end{array}$ & 0.593 \\
\hline $\begin{array}{l}\text { Baseline mean BMI } \\
\qquad(\mathrm{kg} / \mathrm{m} 2)\end{array}$ & 20 & $\begin{array}{c}0.04 \\
(-0.07,0.15)\end{array}$ & 0.440 & 17 & $\begin{array}{c}0.00 \\
(-0.13,0.13)\end{array}$ & 0.979 \\
\hline Baseline mean WC $(\mathrm{cm})$ & 9 & $\begin{array}{c}0.01 \\
(-0.05,0.08)\end{array}$ & 0.677 & 9 & $\begin{array}{c}-0.01 \\
(-0.06,0.03)\end{array}$ & 0.428 \\
\hline
\end{tabular}

BMI: body mass index; WC: waist circumference. 


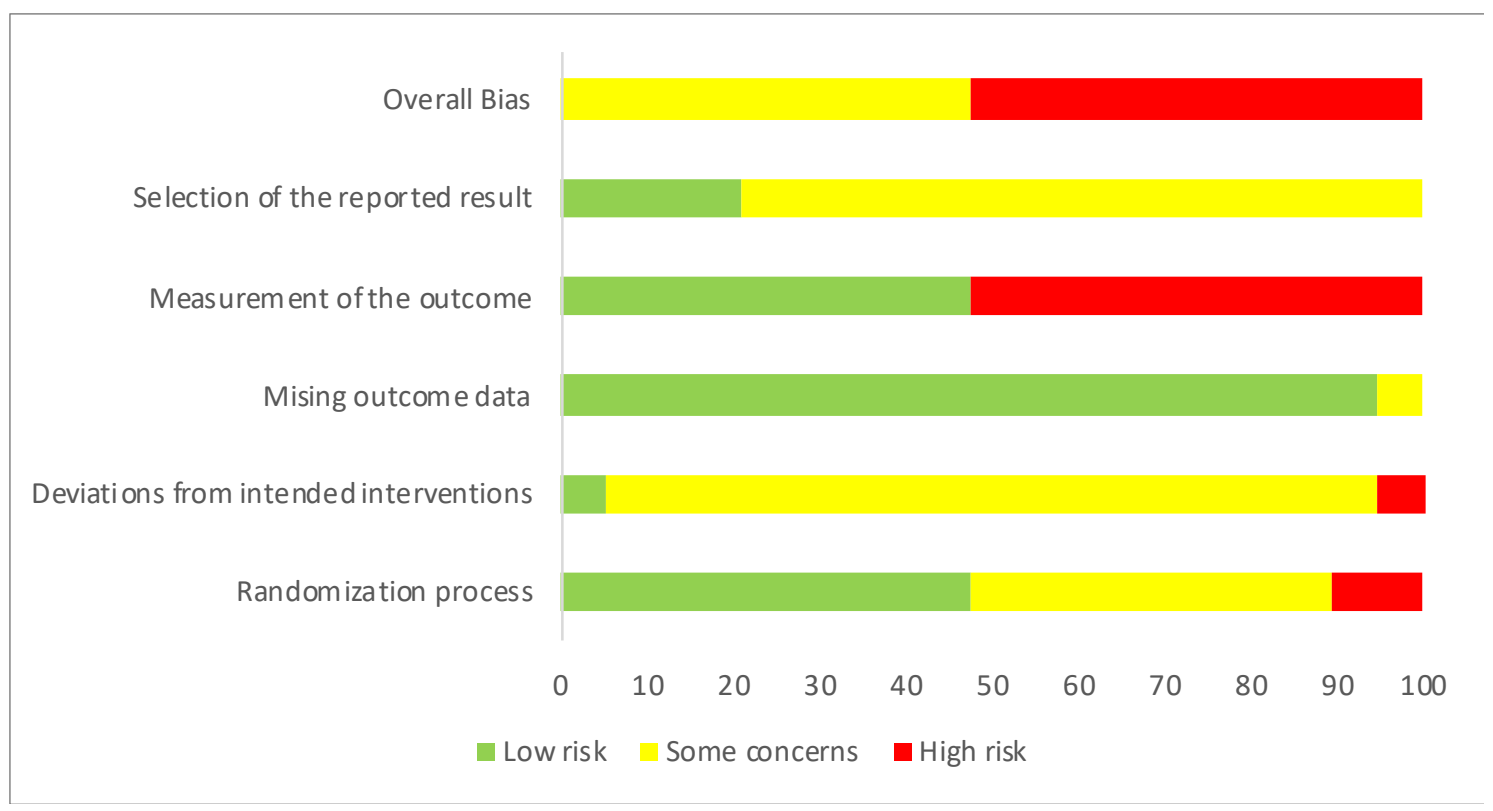

Figure A1. Risk of bias for randomized controlled trials (RCTs) using the Cochrane Collaboration's tool for assessing the risk of bias (RoB2).

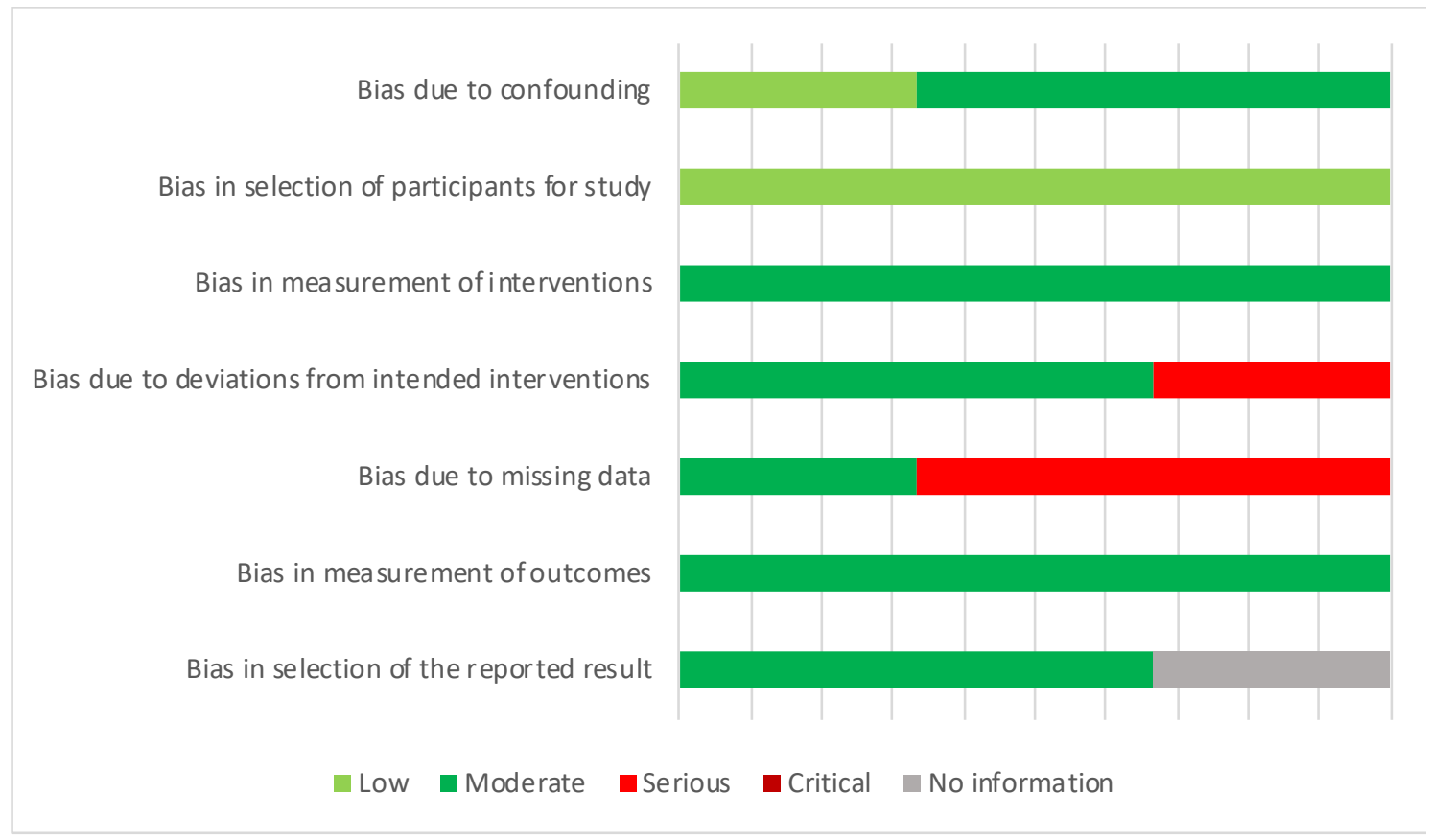

Figure A2. Risk of bias for non-RCTs using the ROBINS-I tool.

\section{References}

1. Mathers, C.; Stevens, G.; Mascarenhas, M. Global Health Risks: Mortality and Burden of Disease Attributable to Selected Major Risks; World Health Organization: Geneva, Switzerland, 2009.

2. Global Burden of Disease Collaborative Network. Global Burden of Disease Study 2015 (GBD 2015) Obesity and Overweight Prevalence 1980-2015; Institute for Health Metrics and Evaluation (IHME): Seattle, WA, USA, 2017.

3. World Health Organization. Global Status Report on Noncommunicable Diseases 2014 (No. WHO/NMH/NVI/15.1); World Health Organization: Geneva, Switzerland, 2014.

4. Kelly, T.; Yang, W.; Chen, C.S.; Reynolds, K.; He, J. Global burden of obesity in 2005 and projections to 2030. Int. J. Obes. 2008, 32, 1431-1437. [CrossRef] 
5. Burke, L.E.; Wang, J.; Sevick, M.A. Self-monitoring in weight loss: A systematic review of the literature. J. Am. Diet. Assoc. 2011, 111, 92-102. [CrossRef] [PubMed]

6. Foster, G.D.; Makris, A.P.; Bailer, B.A. Behavioral treatment of obesity. Am. J. Clin. Nutr. 2005, 82, 230S-235S. [CrossRef] [PubMed]

7. Yu, Z.; Sealey-Potts, C.; Rodriguez, J. Dietary self-monitoring in weight management: Current evidence on efficacy and adherence. J. Acad. Nutr. Diet. 2015, 115, 1931-1933. [CrossRef] [PubMed]

8. Gilmore, L.A.; Duhé, A.F.; Frost, E.A.; Redman, L.M. The technology boom: A new era in obesity management. J. Diabetes Sci. Technol. 2014, 8, 596-608. [CrossRef] [PubMed]

9. DiClemente, R.; Nowara, A.; Shelton, R.; Wingood, G. Need for innovation in public health research. Am. J. Public Health 2019, 109, S117-S120. [CrossRef] [PubMed]

10. WHO. mHealth: New Horizons for Health through Mobile Technologies; WHO Library Cataloguing-in-Publication Data; WHO: Geneva, Switzerland, 2011.

11. Kozak, A.T.; Buscemi, J.; Hawkins, M.A.; Wang, M.L.; Breland, J.Y.; Ross, K.R.; Kommu, A. Technology-based interventions for weight management: Current randomized controlled trial evidence and future directions. J. Behav. Med. 2017, 40, 99-111. [CrossRef] [PubMed]

12. Dounavi, K.; Tsoumani, O. Mobile health applications in weight management: A systematic literature review. Am. J. Prev. Med. 2019, 56, 894-903. [CrossRef]

13. Higgins, J.P.; Green, S. Cochrane Handbook for Systematic Reviews of Interventions. 5.1.0 (Updated March 2011); The Cochrane Collaboration: London, UK, 2011; Available online: https://handbook.cochrane.org (accessed on 1 May 2019).

14. Moher, D.; Liberati, A.; Tetzlaff, J.; Altman, D.G. Preferred reporting items for systematic reviews and meta-analyses: The PRISMA statement. PLoS Med. 2009, 6, e1000097. [CrossRef]

15. Higgins, J.P.; Sterne, J.A.C.; Savovic, J.; Page, M.J.; Hróbjartsson, A.; Boutron, I.; Reeves, B.; Eldridge, S. A revised tool for assessing risk of bias in randomized trials. Cochrane Database Syst. Rev. 2016, 10, $29-31$.

16. Sterne, J.A.C.; Hernán, M.A.; Reeves, B.C.; Savović, J.; Berkman, N.D.; Viswanathan, M.; Henry, D.; Altman, D.G.; Ansari, M.T.; Boutron, I.; et al. ROBINS-I: A tool for assessing risk of bias in non-randomized studies of interventions. BMJ 2016, 355, i4919. [CrossRef] [PubMed]

17. DerSimonian, R.; Kacker, R. Random-effects model for meta-analysis of clinical trials: An update. Contemp. Clin. Trials 2007, 28, 105-114. [CrossRef] [PubMed]

18. Cohen, J. Statistical Power Analysis for the Behavioral Sciences; Lawrence Earlbaum Associates: Hillsdale, MI, USA, 1988; pp. 20-26.

19. Sterne, J.A.C.; Egger, M.; Smith, G.D. Systematic reviews in health care: Investigating and dealing with publication and other biases in meta-analysis. BMJ 2001, 323, 101-105. [CrossRef]

20. Egger, M.; Smith, G.D.; Schneider, M.; Minder, C. Bias in meta-analysis detected by a simple, graphical test. BMJ 1997, 315, 629-634. [CrossRef]

21. Allen, J.K.; Stephens, J.; Himmelfarb, D.C.R.; Stewart, K.J.; Hauck, S. Randomized controlled pilot study testing use of smartphone technology for obesity treatment. J. Obes. 2013, 2013, 151597. [CrossRef]

22. Allman-Farinelli, M.; Partridge, S.R.; McGeechan, K.; Balestracci, K.; Hebden, L.; Wong, A.; Phongsavan, P.; Denney-Wilson, E.; Harris, M.F.; Bauman, A. A mobile health lifestyle program for prevention of weight gain in young adults (TXT2BFiT): Nine-month outcomes of a randomized controlled trial. JMIR Mhealth Uhealth 2016, 4, e78. [CrossRef]

23. Burke, L.E.; Conroy, M.B.; Sereika, S.M.; Elci, O.U.; Styn, M.A.; Acharya, S.D.; Sevick, M.A.; Ewing, L.J.; Glanz, K. The effect of electronic self-monitoring on weight loss and dietary intake: A randomized behavioral weight loss trial. Obesity 2011, 19, 338-344. [CrossRef]

24. Carter, M.C.; Burley, V.J.; Nykjaer, C.; Cade, J.E. Adherence to a smartphone application for weight loss compared to website and paper diary: Pilot randomized controlled trial. J. Med. Internet Res. 2013, 15, e32. [CrossRef]

25. Hartman, S.J.; Nelson, S.H.; Cadmus-Bertram, L.A.; Patterson, R.E.; Parker, B.A.; Pierce, J.P. Technology-and phone-based weight loss intervention: Pilot RCT in women at elevated breast cancer risk. Am. J. Prev. Med. 2016, 51, 714-721. [CrossRef]

26. Hutchesson, M.J.; Callister, R.; Morgan, P.J.; Pranata, I.; Clarke, E.D.; Skinner, G.; Ashton, L.M.; Whatnall, M.C.; Jones, M.; Oldmeadow, C.; et al. A targeted and tailored ehealth weight loss program for young women: The be positive be healthe randomized controlled trial. Healthcare 2018, 6, 39. [CrossRef] 
27. Jospe, M.R.; Roy, M.; Brown, R.C.; Williams, S.M.; Osborne, H.R.; Meredith-Jones, K.A.; McArthur, J.R.; Fleming, E.A.; Taylor, R.W. The effect of different types of monitoring strategies on weight loss: A randomized controlled trial. Obesity 2017, 25, 1490-1498. [CrossRef] [PubMed]

28. Martin, C.K.; Miller, A.C.; Thomas, D.M.; Champagne, C.M.; Han, H.; Church, T. Efficacy of Smart Loss ${ }^{\mathrm{SM}}$, a smartphone-based weight loss intervention: Results from a randomized controlled trial. Obesity 2015, 23, 935-942. [CrossRef] [PubMed]

29. Morgan, P.J.; Callister, R.; Collins, C.E.; Plotnikoff, R.C.; Young, M.D.; Berry, N.; McElduff, P.; Burrows, T.; Aguiar, E.; Saunders, K.L. The SHED-IT community trial: A randomized controlled trial of internet-and paper-based weight loss programs tailored for overweight and obese men. Ann. Behav. Med. 2013, 45, 139-152. [CrossRef] [PubMed]

30. Park, M.J.; Kim, H.S. Evaluation of mobile phone and Internet intervention on waist circumference and blood pressure in post-menopausal women with abdominal obesity. Int. J. Med. Inform. 2012, 81, 388-394. [CrossRef]

31. Ross, K.M.; Wing, R.R. Impact of newer self-monitoring technology and brief phone-based intervention on weight loss: A randomized pilot study. Obesity 2016, 24, 1653-1659. [CrossRef]

32. Sniehotta, F.F.; Evans, E.H.; Sainsbury, K.; Adamson, A.; Batterham, A.; Becker, F.; Brown, H.; Dombrowski, S.U.; Jackson, D.; Howell, D.; et al. Behavioural intervention for weight loss maintenance versus standard weight advice in adults with obesity: A randomised controlled trial in the UK (NULevel Trial). PLoS Med. 2019, 16, e1002793. [CrossRef]

33. Spring, B.; Duncan, J.M.; Janke, E.A.; Kozak, A.T.; McFadden, H.G.; DeMott, A.; Pictor, A.; Epstein, L.H.; Siddique, J.; Pellegrini, C.A.; et al. Integrating technology into standard weight loss treatment: A randomized controlled trial. JAMA Intern. Med. 2013, 173, 105-111. [CrossRef]

34. Stephens, J.D.; Yager, A.M.; Allen, J. Smartphone technology and text messaging for weight loss in young adults: A randomized controlled trial. J. Cardiovasc. Nurs. 2017, 32, 39. [CrossRef]

35. Svetkey, L.P.; Batch, B.C.; Lin, P.H.; Intille, S.S.; Corsino, L.; Tyson, C.C.; Bosworth, H.B.; Grambow, S.C.; Voils, C.; Loria, C.; et al. Cell phone intervention for you (CITY): A randomized, controlled trial of behavioral weight loss intervention for young adults using mobile technology. Obesity 2015, 23, 2133-2141. [CrossRef]

36. Teeriniemi, A.M.; Salonurmi, T.; Jokelainen, T.; Vähänikkila, H.; Alahäivälä, T.; Karppinen, P.; Enwald, H.; Huotari, M.-L.; Laitinen, J.; Oinas-Kukkonen, H.; et al. A randomized clinical trial of the effectiveness of a Web-based health behaviour change support system and group lifestyle counselling on body weight loss in overweight and obese subjects: 2-year outcomes. J. Intern. Med. 2018, 284, 534-545. [CrossRef]

37. Thomas, J.G.; Bond, D.S.; Raynor, H.A.; Papandonatos, G.D.; Wing, R.R. Comparison of Smartphone-Based Behavioral Obesity Treatment With Gold Standard Group Treatment and Control: A Randomized Trial. Obesity 2019, 27, 572-580. [CrossRef] [PubMed]

38. Wang, J.; Cai, C.; Padhye, N.; Orlander, P.; Zare, M. A behavioral lifestyle intervention enhanced with multiple-behavior self-monitoring using mobile and connected tools for underserved individuals with type 2 diabetes and comorbid overweight or obesity: Pilot comparative effectiveness trial. JMIR Mhealth Uhealth 2018, 6, e92. [CrossRef] [PubMed]

39. Wharton, C.M.; Johnston, C.S.; Cunningham, B.K.; Sterner, D. Dietary self-monitoring, but not dietary quality, improves with use of smartphone app technology in an 8-week weight loss trial. J. Nutr. Educ. Behav. 2014, 46, 440-444. [CrossRef]

40. Yon, B.A.; Johnson, R.K.; Harvey-Berino, J.; Gold, B.C.; Howard, A. Personal digital assistants are comparable to traditional diaries for dietary self-monitoring during a weight loss program. J. Behav. Med. 2007, 30, 165-175. [CrossRef] [PubMed]

41. Barbabella, F.; Melchiorre, M.G.; Quattrini, S.; Papa, R.; Lamura, G. How Can eHealth Improve Care for People with Multimorbidity in Europe? World Health Organization, Regional Office for Europe: Copenhagen, Denmark, 2017.

42. Farley, H. Promoting self-efficacy in patients with chronic disease beyond traditional education: A literature review. Nurs. Open 2020, 7, 30-41. [CrossRef] [PubMed]

43. Murray, E.; Burns, J.; Tai, S.S.; Lai, R.; Nazareth, I. Interactive Health Communication Applications for people with chronic disease. Cochrane Database Syst. Rev. 2005, 4, CD004274. [CrossRef] 
44. Elbert, N.J.; van Os-Medendorp, H.; van Renselaar, W.; Ekeland, A.G.; Hakkaart-van Roijen, L.; Raat, H.; Nijsten, T.E.C.; Pasmans, S.G.M.A. Effectiveness and cost-effectiveness of ehealth interventions in somatic diseases: A systematic review of systematic reviews and meta-analyses. J. Med. Internet Res. 2014, 16, e110. [CrossRef]

45. Montesi, L.; El Ghoch, M.; Brodosi, L.; Calugi, S.; Marchesini, G.; Dalle Grave, R. Long-term weight loss maintenance for obesity: A multidisciplinary approach. Diabetes Metab. Syndr. Obes. 2016, 9, 37.

46. Vlahu-Gjorgievska, E.; Mulakaparambil Unnikrishnan, S.; Win, K.T. mHealth applications: A tool for behaviour change in weight management. Stud. Health Technol. Inform. 2018, 252, 158-163.

47. Robbins, R.; Krebs, P.; Jagannathan, R.; Jean-Louis, G.; Duncan, D.T. Health app use among US mobile phone users: Analysis of trends by chronic disease status. JMIR Mhealth Uhealth 2017, 5, e197. [CrossRef]

48. Gordon, W.J.; Landman, A.; Zhang, H.; Bates, D.W. Beyond validation: Getting health apps into clinical practice. NPJ Digit. Med. 2020, 3, 1-6. [CrossRef] [PubMed]

49. Smith, A.C.; Thomas, E.; Snoswell, C.L.; Haydon, H.; Mehrotra, A.; Clemensen, J.; Caffery, L.J. Telehealth for global emergencies: Implications for coronavirus disease 2019 (COVID-19). J. Telemed. Telecare 2020. [CrossRef] [PubMed]

(C) 2020 by the authors. Licensee MDPI, Basel, Switzerland. This article is an open access article distributed under the terms and conditions of the Creative Commons Attribution (CC BY) license (http://creativecommons.org/licenses/by/4.0/). 